\title{
Photonic crystal laser sources for chemical detection
}

\author{
Marko Lončara) and Axel Scherer \\ Department of Electrical Engineering, California Institute of Technology, MS 136-93, Pasadena, \\ California 91125 \\ Yueming Qiu \\ In Situ Technology and Experiments Systems Section, Jet Propulsion Laboratory, California \\ Institute of Technology, MS 302-306, Pasadena, California 91109
}

(Received 3 March 2003; accepted 23 April 2003)

\begin{abstract}
We have realized photonic crystal lasers that permit the introduction of analyte within the peak of the optical field of the lasing mode. We have explored the design compromises for developing such sensitive low-threshold spectroscopy sources, and demonstrate the operation of photonic crystal lasers in different ambient organic solutions. We show that nanocavity lasers can be used to perform spectroscopic tests on femtoliter volumes of analyte, and propose to use these lasers for high-resolution spectroscopy with single-molecule sensitivity. (c) 2003 American Institute of Physics. [DOI: 10.1063/1.1586781]
\end{abstract}

The construction of compact spectroscopic tools for the optical analysis of ultrasmall $\left(<10^{-15}\right.$ liter) sample volumes remains an important goal in the development of integrated microfluidics systems. Miniaturization of appropriate light sources and detectors can enable very compact and versatile "laboratory on a chip" devices, in which many analytical functions can be monolithically combined. One of the device integration platforms which is ideally suited to enable such integration of ultrasmall and efficient optical components is the membrane-based planar photonic crystal, ${ }^{1-3}$ defined in high refractive index contrast materials by standard lithography and semiconductor fabrication processes. High-quality optical cavities with mode volumes far below a cubic wavelength have already been demonstrated, and can be used to obtain very high optical field intensities from ultrasmall laser sources. ${ }^{4-10}$ Here, we describe the application of such planar photonic crystal lasers optimized for chemical sensors, with high spectral resolution and excellent sensitivity to changes in the absorption or refractive index of their surrounding. Our porous cavity design permits the introduction of analyte directly into the high optical field of the laser cavity, and, due to the ultrasmall mode volume of our lasers, permits the sensitivity to optical changes within sub-atoliter volumes. The introduction of absorbing or fluorescing molecules into such cavities is expected to have a large influence on the optical signature, and the high fields obtained in the cavity can be used for spectroscopy of the cavity contents. This will enable the sensing and analysis of individual organic molecules or self-assembled quantum dots, and offers a unique opportunity to achieve strong interaction between light and molecules on a nanoscale level.

The cavity geometry that we have chosen for our chemical sensors is based on a single-defect triangular lattice planar photonic crystal. In addition to the introduction of a smaller hole to define the optical cavity, we also introduce a fractional edge dislocation ${ }^{4}$ by extending the length of one of the rows of holes to break the symmetry of the optical cavity.

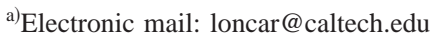

In our previous publications, we have experimentally described the advantages of such a structure, and shown that it supports modes with both high-quality factors ${ }^{5}(Q)$ and small mode volume ${ }^{6}\left(V_{\text {mode }}\right)$. When defined within InGaAsP membranes, low-threshold room-temperature lasers have also been defined. ${ }^{7}$ The cavity design is shown in Fig. 1 and it can be seen that the energy of the mode is mostly confined to the central defect hole. In order to increase the interaction between light and the material within that hole, a larger defect hole diameter is preferred. However, increasing this hole reduces the gain provided by the light-emitting quantum wells within the laser cavity and thereby increases the threshold of our laser. Therefore, a trade off between the optical overlap with the analyte and the optical gain is needed, and we have addressed this problem using three-dimensional finitedifference time-domain modeling. The thickness of photonic crystal slab with a refractive index of 3.4 was $d=0.75 a$, where $a$ is the lattice parameter of the photonic crystal, and the size of the holes in the photonic crystal mirror surrounding the cavity was $r=0.3 a$. A single-defect donor cavity in a triangular lattice photonic crystal without the fractional edge dislocation is known to support two doubly degenerate, linearly polarized, dipole modes ${ }^{8}$ with rather modest quality factors of several hundred. However, as we stretch the photonic crystal lattice by introducing a fractional dislocation $(p)$, these modes start to interact, the degeneracy between them is lifted, and the quality factor of one of these modes is
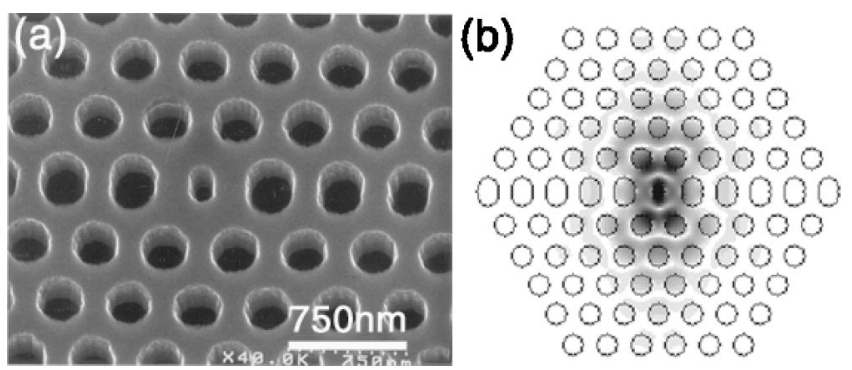

FIG. 1. (a) Scanning electron micrograph and (b) calculated field distribution of a photonic nanocavity laser sensor. 

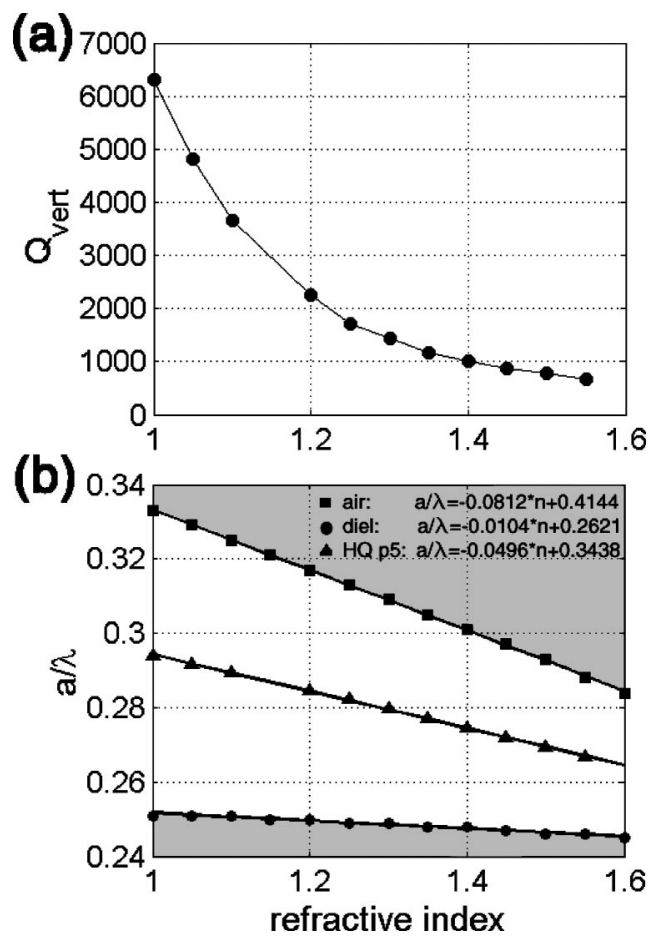

FIG. 2. Dependence of the (a) $Q$ factor and (b) eigenfrequency of cavity resonance on the refractive index of the analyte introduced in the cavity with $p / a=25 \%$.

increased to over $6000 .^{11}$ It is of interest to determine the change in this $Q$ as the cavity is backfilled with reagents. From Fig. 2(a), we observe that the highest $Q$ in the modeled cavity design occurs at an ambient refractive index of $n$ $=1$, and this value deteriorates as the refractive index of the ambient surrounding the photonic crystal cavity is increased. This decrease in $Q$ is a result of the weaker vertical confinement of light by total internal reflection, and can be compensated for by increasing the thickness of the photonic crystal slab. Another important figure of merit for evaluating the performance of our laser spectrometer is the gain provided by the active material to the lasing mode. As the defect hole diameter is decreased and the amount of dislocation is increased, we can expect a better overlap between the optical cavity mode and the quantum wells, and a decrease in the laser threshold. However, it is important in spectroscopy applications that the defect hole is large in order to obtain the desired interaction between the optical cavity field and the reagent. Therefore, we have chosen $r_{\mathrm{def}}=0.15 a$ and $p$ $=0.25 a$ as a good compromise for our initial laser sensor design. It is interesting to note that the frequency of the resonant mode, as well as the band edges of the photonic crystal mirror, depend linearly on the refractive index of the analyte [Fig. 2(b)]. From linear fits of the dependence of the resonant frequency on the refractive index of the analyte $\left(n_{\text {analyte }}\right)$, we can estimate the sensitivity of the cavity, and we predict that the wavelength shift of the resonance should be approximately $\Delta \lambda \approx 266 \cdot \Delta n$, where $\Delta n$ is the change in refractive index. The simplest method of optically sensing ambient material uses wavelength shifts in the laser spectrum when the laser is immersed into a solution or exposed to a material to measure its refractive index. In this method, the sensitivity of the sensor depends on the smallest change in refractive index that can be optically detected. In passive Downloaded 18 Dec 2005 to 131.215.240.9. Redistribution subjec
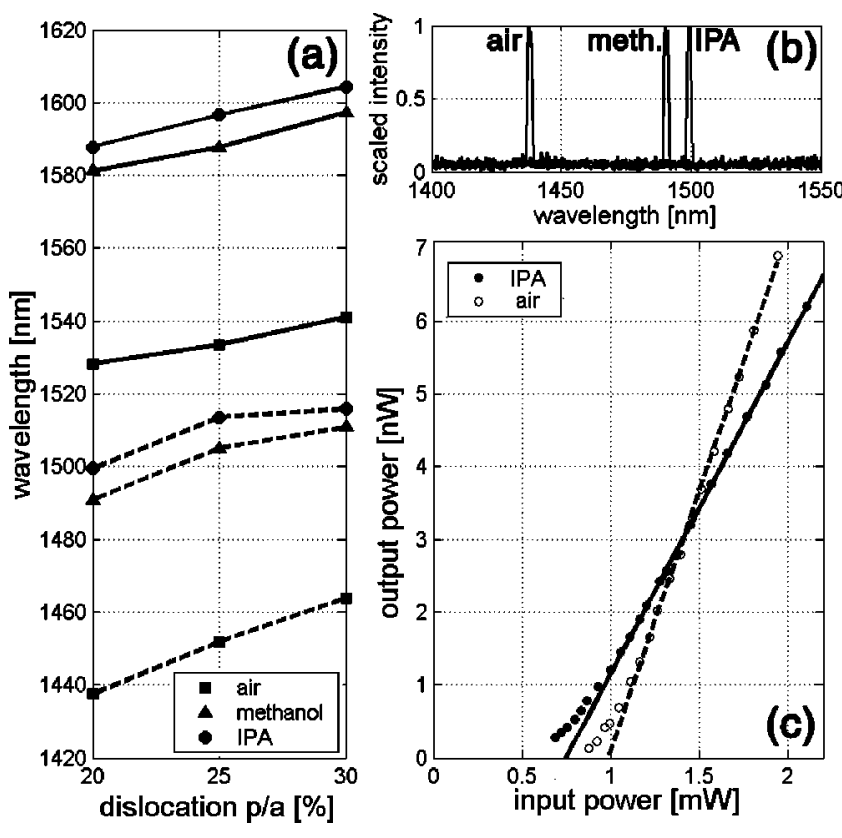

FIG. 3. (a) Sensor response measured from six nanocavities with lattice parameters of $446 \mathrm{~nm}$ (dashed lines) and $460 \mathrm{~nm}$ (solid lines) as a function of $p / a$ dislocation parameters. The three curves correspond to laser wavelength with air, methanol, and IPA backfilled into the cavity. (b) Spectrum change and (c) threshold curve change of laser before and after filling with IPA.

devices, this is related to the width of the cavity resonance peak, which in turn is determined by cavity quality $Q$. If we assume that our cavity is embedded in a typical polymer ( $n$ $\approx 1.4$ ), a wavelength shift that is still observable from cavity $\left(Q_{n=1.4}=1000\right)$ is $\Delta \lambda \approx 1.55 \mathrm{~nm}$, what corresponds to change in the refractive index of $\Delta n \sim 0.0056$. On the other hand, once we introduce optical gain into the cavity, as in the case of the proposed laser spectrometer, the linewidth of emission is significantly narrowed, and therefore much higher sensitivities of $\Delta n<0.001$ can be measured even in cavities with modest $Q$ factors.

To demonstrate this, photonic crystal nanolasers were fabricated from InGaAsP quantum well material. Optical gain was provided by four $9 \mathrm{~nm}$ thick, compressively strained quantum wells, placed in the center of a $330 \mathrm{~nm}$ thick InGaAsP slab. The emission from the quantum wells was in the range of $1300 \mathrm{~nm}<\lambda<1600 \mathrm{~nm}$, with a maximum at $1550 \mathrm{~nm}$, and these were embedded within a free standing membrane, patterned with a photonic crystal lattice as shown in Fig. 1. The precise emission wavelength could be controlled either by scaling the lattice parameter, or by changing the size of the defect hole introduced into the lattice to form the cavity. The structures were tested using a microphotoluminescence approach, and were optically pumped at room temperature with $30 \mathrm{~ns}$ pulses of $3 \mu \mathrm{s}$ periodicity $\left(\lambda_{\text {pump }}=830 \mathrm{~nm}\right)$. Further details of the fabrication procedure and experimental method can be found in Ref. 7 . To test the influence of a change in the ambient refractive index on the laser spectrum of a cavity, we have immersed our photonic crystal lasers in glass beakers containing isopropyl alcohol (IPA) and methanol. Figure 3 shows position of the resonances from six different lasers after immersion in air, IPA, and methanol. It can be seen that wavelength shifts of up to $67 \mathrm{~nm}$ can be observed when a cavity is immersed in to AIP license or copyright, see http://apl.aip.org/apl/copyright.jsp 

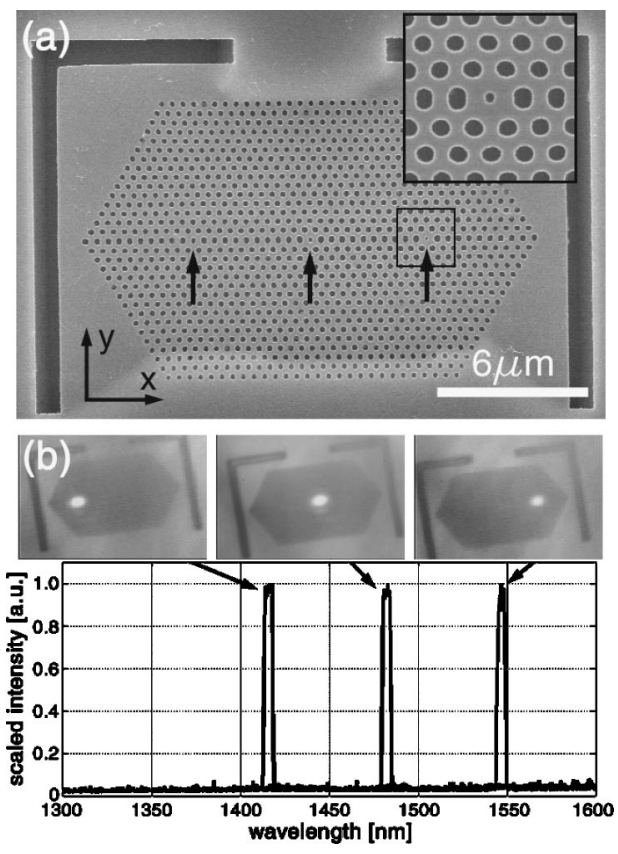

FIG. 4. (a) Fabricated structure consists of three cavities integrated within the same photonic crystal mirror. Defect holes are indicated by arrows, and their size increases from the right- to left-hand side. (b) Resonances detected in each cavity. Mode experience blueshift as the size of defect hole increases. Positions of pump beam are shown.

IPA. This redshift corresponds to a change in refractive index from 1.0 to 1.377 , and yields a roughly $1 \mathrm{~nm}$ spectral shift for a 0.0056 change in refractive index. When IPA is replaced with methanol $(n=1.328)$, the laser resonance experiences a blueshift of $\sim 9 \mathrm{~nm}$, while our numerical analysis predicts a shift of $\sim 13 \mathrm{~nm}$ (Fig. 2). The discrepancy can be attributed to the differences between modeled and experimentally characterized structures. We have also investigated the dependence of the cavity resonance wavelength on the lithographic laser geometry, particularly the lattice constant and the dislocation in the photonic crystal cavity. Resonances experience redshifts of $\sim 80 \mathrm{~nm}$ when the periodicity is changed from $a=446 \mathrm{~nm}$ (dashed lines) to $a=460 \mathrm{~nm}$ (solid lines) as indicated in Fig. 3(a). This lithographic tuning of the emission wavelength can be used to ensure an overlap of the cavity resonance peak with the InGaAsP quantum well emission gain curve even when the cavities are immersed in a reagent. Laser threshold curves before and after immersion into alcohol are also presented in Fig. 3. After immersion, the laser threshold power for the cavity measured was reduced since the emission wavelength was shifted to match the maximum gain of the quantum wells. However, the differential quantum efficiency of the immersed cavity is slightly lower, which may reflect the lower laser cavity $Q$ after immersion.

We have also tested structures with different defect hole sizes $\left(r_{\mathrm{def}} / a\right)$ within the same photonic crystal slab in order to explore the integration multiwavelength photonic crystal lasers with lithographically predetermined spectra. These devices are particularly interesting as compact multiwavelength light sources, but are also useful if many reactions have to be monitored at the same time. Individual reactions can be observed in laser cavities which have predetermined spectral signatures, and optically read by observing changes in the Downloaded 18 Dec 2005 to 131.215.240.9. Redistribution subject collective spectrum of a multiwavelength laser array. In Fig. 4 , we show both the structure and accompanying spectra of three optical cavities fabricated within a common photonic crystal slab with $a=446 \mathrm{~nm}$ and $r=134 \mathrm{~nm}$. The sizes of the defect holes which define the optical cavities were varied from $r_{\text {small }}=74 \mathrm{~nm}, r_{\text {mid }}=85 \mathrm{~nm}$, and $r_{\text {big }}=97 \mathrm{~nm}$, and a detailed view of one of the cavities is shown in the inset of Fig. 4. The distance between the three cavities is 10 lattice periods or $\sim 4.5 \mu \mathrm{m}$. To measure these lasers, the cavities were pumped individually, and well-confined spectra were obtained from each of these cavities. The lasing wavelength of these cavities could be tuned from $1420 \mathrm{~nm}$ (for $r_{\text {big }}$ ) to 1550 $\mathrm{nm}$ (for $r_{\text {small }}$ ). Since InGaAsP can serve both as a laser source material as well as a material for light detection, it is possible to develop compact integrated spectroscopy systems for chemical and biological analysis, and filtered photonic crystal nanocavity detectors can be included to monitor the output of adjacent laser diodes.

In conclusion, high- $Q$ laser cavities with small mode volumes provide us with opportunities for integrated optical spectroscopy systems. We have demonstrated such lasers and show that shifts in the refractive index of the ambient surrounding the laser cavities can be measured by observing the laser spectrum. Small changes in refractive index can be detected within samples of femtoliter volumes, and such devices can be integrated into large arrays to permit the analysis of many reagents at the same time. Our laser devices are intended for biochemical sensing by backfilling the defect hole in our photonic crystal cavity with reagent. Absorption measurements and Raman spectroscopy are other very interesting methods for the analysis of chemicals, which can benefit from the very high optical fields available in a nanolaser cavity.

The authors would like to thank Luka Lončar, Tomoyuki Yoshie, David Barsic, and Guy DeRose for discussions and help in the lab. This work was supported by the NSF under Grant Nos. BES-0119493 and ECS-9912039, AFOSR under Grant No. F49620-01-6-0497, and DARPA under Grant No. MDA972-00-1-0019. One of the authors (Y.Q.) acknowledges the partial support from the Cross Enterprise Technology Development Program at the Jet Propulsion Laboratory (under a contract with NASA).

${ }^{1}$ S. G. Johnson, S. H. Fan, P. R. Villeneuve, J. D. Joannopoulos, and L. A. Kolodziejski, Phys. Rev. B 60, 5751 (1999).

${ }^{2}$ T. Baba, A. Motegi, T. Iwai, N. Fukaya, Y. Watanabe, and A. Sakai, IEEE J. Quantum Electron. 38, 743 (2002).

${ }^{3}$ S. Noda, A. Chutinan, and M. Imada, Nature (London) 407, 608 (2000).

${ }^{4}$ J. Vučković, M. Lončar, H. Mabuchi, and A. Scherer, Phys. Rev. E 65, 016608 (2001).

${ }^{5}$ T. Yoshie, J. Vučković, A. Scherer, H. Chen, and D. Deppe, Appl. Phys. Lett. 79, 4289 (2001).

${ }^{6}$ K. Okamoto, M. Lončar, T. Yoshie, A. Scherer, Y. Qiu, and P. Gogna, Appl. Phys. Lett. 82, 1676 (2003).

${ }^{7}$ M. Lončar, T. Yoshie, A. Scherer, P. Gogna, and Y. Qiu, Appl. Phys. Lett. 81, 2680 (2002).

${ }^{8}$ O. Painter, J. Vučković, and A. Scherer, J. Opt. Soc. Am. B 16, 275 (1999).

${ }^{9}$ H. G. Park, J. K. Hwang, J. Huh, H. Y. Ryu, and Y. H. Lee, Appl. Phys. Lett. 79, 3032 (2001).

${ }^{10}$ H. Y. Ryu, S. H. Kim, H. G. Park, J. K. Hwang, and Y. H. Lee, Appl. Phys. Lett. 80, 3883 (2002).

${ }^{11}$ M. Lončar, T. Yoshie, Y. Qiu, P. Gogna, and A. Scherer (unpublished). 